\title{
Nanomedical Platform for Drug Delivery
}

\section{Naga Anusha $\mathbf{P}^{1 *}$ and Aliya Siddiqui ${ }^{2}$}

${ }^{1}$ Department of Biotechnology, Sri Y.N. College, Andhra University, Narsapur, India

${ }^{2}$ Department of Biotechnology, Chaitanya P.G.College, Kakatiya University, Warangal, India

\section{Abstract}

Nanoparticles hold tremendous potential as an effective drug delivery system. The developments in nanotechnology for drug delivery laid platform for nanomedicine. To achieve efficient drug delivery it is important to understand the interactions of nanomaterials with the biological environment. This rapidly growing field allows cross-disciplinary researchers the opportunity to design and develop multifunctional nanoparticles in the form of nanotubes that can target, diagnose, and treat diseases such as cancer. Here I discussed about the engineering of nanotubes which targets drug delivery, barriers of drug delivery and advanced drug delivery systems.
\end{abstract}

Keywords: Nanotechnology; Nanomedicine; Nanotubes; Carbon nanotubes; Fullerenes; Dendrimers; Gold nanoparticles; Silver nanoparticles; Drug Delivery; Blood Brain Barrier

Abbreviations:NP- NanoParticles; CNT- Carbon NanoTubes; BBBBlood Brain Barrier; PLA- Poly Lactic Acid; PGA- Poly Glycolic Acid; MSN- Mesoporous silica Nanoparticles; CNS- Central Nervous System

\section{Introduction}

Nanomedicine is the application of nanotechnology which is used to prevent and treatment of diseases in human body at molecular scale. Recent years have witnessed the unpredicted growth of research and applications in nanotechnology [1]. Nanotechnology as applied to medicine will bring significant advances in diagnosis and treatment of disease. Nanomedicine includes several distinct application areas: Drug delivery, drugs and therapies, in vivo imaging, in vitro diagnostics, biomaterials, and active implants [2,3]. In these fields, Nanomedicine has seen a surge in research activity over the past decade. The various drives of Nanomedicine include direct target of diseased tissue, the early diagnosis of cancer, transport of drugs through Blood-Brain Barrier and the development of implant materials with long life span [4]. Nanoparticles (NPs) use their optical scattering properties for imaging and diagnostics and their photo thermal properties for various types of therapies through the generation of heat, vapor bubbles and acoustic waves [5].

\section{Nanoparticles and Drug Delivery}

Nanotechnology based drug delivery uses a variety of nanovectors including liposomes, micelles, dendrimers, protein nanoparticles, ceramic nanoparticles, polymeric nanoparticles and metallic nanoparticles [6]. Nanoparticles, a unique subset of the broad field of nanotechnology, include type of particle with at least one dimension of less than 500 nanometers [7]. Nanoparticles play an important role in a wide variety of fields advanced medicine, pharmaceuticals, and environmental detection and monitoring. In medical field Nanoparticles play important role in drug delivery [8]. Nanoparticles are also used in targeting antibody $[9,10]$. Over the years, a variety of natural and synthetic polymers have been explored for the preparation of nanoparticles, of which Poly Lactic Acid (PLA), Poly Glycolic Acid (PGA), and their copolymers (PLGA) have been extensively investigated because of their biocompatibility and biodegradability. Nanoparticles act as potential carries for several classes of drugs such as anticancer agents, antihypertensive agents, immunomodulators, and hormones; and macromolecules such as nucleic acids, proteins, peptides, and antibodies [11]. Paclitaxel is an anticancer drug used for lung, breast and ovarian tumors. It is generally administered as a mixture with poly (oxyethylene) castor oil (Cremophor EL) and dehydrated ethanol which were proved to cause side effects. Attempts are being made to deliver this drug through nanoparticles [12]. The options available for preparation have increased with advances in traditional methods, and many novel techniques for preparation of drug-loaded nanoparticles are being developed and refined [13]. The performance of nanoparticles in vivo is influenced by morphological characteristics, surface chemistry, and molecular weight. Careful design of these delivery systems with respect to target and route of administration may solve some of the problems faced by new classes of active molecules [14]. The primary goals of nanoparticle based drug delivery are [15]:

- Specific drug delivery and targeting

- Maintaining therapeutic effects and decrease in toxicity

- Biocompatibility

- Fast development of new safe medicines

\section{Engineering of Nanotubes}

Engineered nanoparticles have the potential to revolutionize the diagnosis and treatment of many diseases; for example, by allowing the targeted delivery of a drug to particular subsets of cells [16]. Many parameters have to be followed in preparation of nanotubes particularly for drug delivery particularly biodegradation of nonmaterial, less toxicity [17]. Drug delivery through particular target can be done by using carbon nanotubes, fullerenes, Dendrimers, gold nanoparticles, silicon nanotubes [18]. These were proven to be efficient source of drug deliver. Small size of nanoparticles leads to particle-particle aggregation, making physical handling of nanoparticles difficult in liquid [19] and dry powder forms. To solve these limitations, the production of spray-dried powders containing nanoparticles, Which are localized as polymeric nanoparticles of sufficiently small dimensions avoid mucociliary and phagocytic clearance until the particles have delivered their therapeutic payload [20].

*Corresponding author: Naga Anusha Puvvada, Department of Biotechnology, Sri Y.N. College, Narsapur, India; E-mail: anushanpuvvada@gmail.com

Received November 01, 2011; Accepted December 03, 2011; Published December 05, 2011

Citation: Naga Anusha P, Siddiqui A (2011) Nanomedical Platform for Drug Delivery. J Nanomedic Nanotechnol 2:122. doi:10.4172/2157-7439.1000122

Copyright: (C 2011 Naga Anusha P, et al. This is an open-access article distributed under the terms of the Creative Commons Attribution License, which permits unrestricted use, distribution, and reproduction in any medium, provided the original author and source are credited. 
Carbon nanotubes: Carbon nanotubes (CNTs) are allotropes of carbon with a nanostructure that can have a length-to-diameter ratio greater than 1,000,000. These cylindrical carbon molecules have novel properties that make them potentially useful in many applications in nanotechnology. Their unique surface area, stiffness, strength and resilience have led to much excitement in the field of pharmacy [21]. Nanotubes offer many advantages in drug delivery mainly focusing on improved safety and efficiency of the drugs: for example providing targeted delivery of drugs, improving bioavailability, extending drug or gene effect of drugs, tissue and improving the stability of therapeutic agents against chemical/enzymatic degradation [22]. Carbon nanotubes based drug delivery has great promise for cancer ablation. Their use as drug delivery scaffolds and substrates for vaccines has already been demonstrated [23]. CNT functionalized with bioactive moieties are particularly suited for targeted drug delivery. In fact, not only they become less toxic but also exhibit a high propensity to cross cell membranes. CNTs can be functionalized by attaching biological molecules such as lipids, proteins, biotins etc to them, through this it is possible to solubilise and disperse carbon nanotubes in water, thus opening the path for their facile manipulation and processing in physiological environments.

Fullerenes: Fullerenes are a new class of compounds with potential uses in biology and medicine and many insights were made in the knowledge of their interaction with various biological systems. Fullerenes tend to form nano aggregates in aqueous solutions [24]. Fullerene (C60) derivatives have been extensively studied for a variety of medical applications, which include neuroprotective agents, HIV-1 protease inhibitors, and photosensitizers for photodynamic therapy, MRI contrast agents, and radiopharmaceuticals. The first part of this work is dedicated to the development of a new application of C60 as a slow-release system for the liposome aerosol delivery of lipophilic chemotherapeutics to lung and breast cancer [25]. TaxolRTM (Paclitaxel), one of the most active anticancer drugs in clinical use, has shown significant potential for treatment of lung cancer when delivered by the liposome aerosol method.

Dendrimers: Dendrimers are built from a starting atom, such as nitrogen, after a repeating series of chemical reactions, carbon and other elements was added into it; produce a spherical branching structure called dendritic polymer which provide well defined nano structure for drug delivery, targeting for particular receptor specific site. Poly AMido-AMine (PAMAM) dendrimers were used for drug delivery [26].

Gold Nanoparticles: Gold is used for nanoparticle applications because it is unreactive and is not sensitive to air or light. However gold does like to form bonds with it and for this reason their surfaces have to be covered with a layer of protective molecules, for example sulfur compounds. Gold nanoparticles (AuNPs) provide non-toxic carriers for drug and gene delivery applications that provide a useful complement to more traditional delivery vehicles as well as in diagnosis [27]. Gold nanoparticles have property of converting allowed radiofrequency into high frequency is known as 'Quantum Dot' property and can be used for destroying cancer cells $[28,29]$. Their combination of low inherent toxicity, high surface area and tunable stability provides them with unique attributes that should enable new delivery strategies [30].

Silicon Nanotubes: Mesoporous silica-based materials show number of attractive features for biomedical applications, such as stable mesoporous structures, large surface areas, tunable pore sizes and volumes, good biocompatibility, and well-defined surface properties available for further fictionalizations [31,32]. The structure, morphology, size, and surface properties of MSNs have been found to be facilely tunable for the purposes of drug loading, controlled drug release and delivery, and multifuctionalization. These MSNs have an average diameter less than $80 \mathrm{~nm}$ and exhibit well ordered porous structure and with aqueous stability.

Apart from these there were some natural polymers that include Starch, chitosan [33], alginate, and dextrans. These polymers are applied as colloidal particles of size $10 \mathrm{~nm}-1 \mu \mathrm{m}$ termed nano-particles. In this system, the drug to be delivered could be dispersed within the polymeric matrix or adsorbed on the surface of the carrier in which case they are called nano-spheres or it could be encapsulated within a core surrounded by polymeric membrane and are known as nanocapsules [34].

\section{Targets for Drug Delivery}

One of the major challenges in drug delivery is to carry the drug at the place where it is needed and to avoid potential side effects on non diseased organs. After reaching the targeted tissue, drugs should have the ability to selectively kill diseased cells without affecting normal cells with a controlled release mechanism of the active form [35]. These basic strategies are also associated with improvements in patient survival and quality of life by increasing the intracellular concentration of drugs and reducing dose-limiting toxicities simultaneously. Increasingly, nanoparticles seem to have the potential to satisfy both of these requirements for effective drug carrier systems [36].

Cellular \& Intracellular Targets: Intracellular targeting refers to the delivery of therapeutic agents to specific compartments or organelles within the cell. The therapeutic agent could be a low molecular weight drug or a macromolecule like protein or DNA. There are number of ways in which therapeutic drug can be delivered into target cell. This may be by simple diffusion or it may involve complex cellular machinery [37]. The major route of intracellular therapeutic uptake is through endocytosis [38]. The endocytic processes described to date include fluid phase pinocytosis, clathrin-coated pit endocytosis, caveoli, macropinocytosis and phagocytosis. All the endocytic processes are energy dependent. Thus, endocytosis is at least partially inhibited either at low temperatures or by cellular ATP depletion using metabolic inhibitors such as sodium azide (inhibits oxidative phosphorylation) and 2- deoxyglucose (inhibits glycolysis). The intra cellular drug delivery involves Endo-Lysosomal targeting in which anti bacterial and antiparasitic agents to lysosomes and phagolysosomes [39]. The need for antibiotics with greater intracellular efficacy led to the use of endocytosable drug carriers such as liposomes and nanoparticles, which mimic the entry path of the bacteria by penetrating into phagosomes or lysosomes [40]. Another type of intracellular drug delivery involves cytoplasmic delivery and this is so because the receptors for steroids were present in the cytoplasm and hence, by delivering the drug at or near its receptor site, one could achieve better therapeutic effect and at the same time reduce the undesirable side effects [41]. A number of gene carriers that escape the endo-lysosomal compartment have been found to be good transfecting agents. Viruses and peptide toxins use a fusogen peptide to cross the endosomal membrane and reach the cytosol. Similarly, non-viral vectors such as cationic lipids and polycations protect the DNA by either retarding the transfer of DNA from endosomes to lysosomes or destabilize the endo-lysosomal membranes [42]. The next type of targeting is nuclear targeting. The nuclear targeting of macro molecules has been extensively studied and has been used for delivering therapeutic agents specifically into nucleus [43]. Main application of nuclear targeting has been to deliver 
plasmid DNA for improving the efficiency of gene expression. In nondividing cells, the rate-limiting barrier to efficient gene transfection is the nuclear membrane [44]. Agents such as Trichostatin A that inhibit the nuclear export of the transcription factor NF-kB have been shown to have potential anti-tumor efficacy. Mitochondrial-targeted drug delivery is also important for the delivery of antioxidant molecules to mitochondria for preventing oxidative damage $[45,46]$. Oxidative damage causes non-specific damage to lipids, proteins and DNA and many respiratory chain proteins, especially iron-sulfur proteins, are particularly susceptible to oxidative damage leading to a decline in the efficiency of oxidative phosphorylation. Mitochondrial oxidative damage increases in a wide variety of diseases including ischemiareperfusion injury and neurodegenerative diseases, contributing to their pathophysiology by disrupting mitochondrial function.

Brain - the ultimate Target: Nanoparticles can also be used for getting drugs into brain. The Blood- Brain Barrier is an endothelial interfaces with tight junctions, which unable the entry of many drugs to brain . However, nanoparticle drug delivery is particularly useful for disorders of Central nervous System because some nano particles are able to cross BBB. Often they can deliver drugs directly into CNS. Transport of NPs across the BBB has been characterized similarly too many of the known transport mechanisms described for other drugs. Passive diffusion at the $\mathrm{BBB}$ occurs when a drug dissolves in the lipid membrane of cerebrovascular endothelial cells and then released into the brain [47]. Passive diffusion depends on: (1) the lipophilicity of the drug, (2) charge, (3) concentration gradient, (4) molecular weight, and (5) the degree of protein binding. Solid lipid nanoparticles have also been proposed to be useful in delivering various drugs across the blood brain barrier [48,49]. Bioactive molecules like antisense oligonucleotides loaded in solid lipid nanoparticles were claimed to penetrate the blood brain barrier and are suggested for use in the treatment of various cerebral diseases [50].

\section{Advanced Drug Delivery System}

Controlled drug-delivery strategies have made a dramatic impact in medicine. In general, controlled-release polymer systems deliver drugs in the optimum dosage for long periods, thus increasing the efficacy of the drug, maximizing patient compliance and enhancing the ability to use highly toxic, poorly soluble or relatively unstable drugs. Nanoscale materials can be used as drug delivery vehicles to develop highly selective and effective therapeutic and diagnostic modalities.

Liposome mediated drug delivery: Designing adequate drug carriers has long been a major challenge for those working in drug delivery. Since drug delivery strategies have evolved for mucosal delivery as the outstanding alternative to parenteral administration, many new drug delivery systems have been developed which evidence promising properties to address specific issues [51]. Colloidal carriers, such as nanoparticles and liposomes, have been referred to as the most valuable approaches, but still have some limitations that can become more inconvenient as a function of the specific characteristics of administration routes [52]. The lipid/chitosan nanoparticle complexes are expected to protect the encapsulated drug from harsh environmental conditions, while concomitantly providing its controlled release [53]. To prepare these assemblies, two different strategies have been applied: one focusing on the simple hydration of a previously formed dry lipid film with a suspension of chitosan nanoparticles, and the other relying on the lyophilization of both basic structures (nanoparticles and liposomes) with a subsequent step of hydration with water [54]. Liposomal nanoparticle therapeutics containing cytotoxic agents, nanotechnology-enabled delivery of GMPgrade WHI-P131 showed potential treatment for breast cancer and shown to be more effective and less toxic anti-cancer treatment strategies due to their improved pharmacokinetics, reduced systemic toxicity, and increased intratumoral/intracellular delivery [55].

Nanoparticles in gene delivery: Gene delivery systems are an important area in the field of genetic nano medicine [56]. Nanoparticle mediated gene delivery had recently emerged as a promising tool for gene therapy strategies. Substantial progress has been made in binding DNA to nanoparticles and controlling the behavior on binding DNAs to nanoparticles as well as on non-viral gene delivery using polyethylenimine-coated magnetic nanoparticles. Currently there were three different gene delivery systems for transferring gene inserts into host cell [57]. They include viral gene delivery, nucleoacid electroporation and nucleic acid transfection. Gene delivery through viral vector methods is proven to be more efficient but it may insert viral vector nucleic acid sequence into host genome, causing unwelcome effects such as inappropriate expression of deleterious genes. Magnetic nanoparticles were the most efficient gene delivery vectors tested. Many researchers have developed magetofection methods. They modified the surface of iron oxide- based MNPs to increase transfection efficiency and to reduce cytotoxicity [58]. Cells incubated with these particles showed no visible signs of toxicity (blebbing, apoptosis, etc.), even though the particles are made of iron. The MNP offer the most promise of the nanoparticles evaluated [59].

Apart from medical field nanotechnology plays important role in studying electron-photon interactions, protein strategies [60] creation of nano structure films and interactions [61]. Nanobiosensors were being developed that sense the level of body fluids like blood $[62,63,64,65]$.

\section{Conclusion}

Targeted drug delivery has become critical wake in the availability of therapeutic agents. Nanoparticles formulated from different polymers and other nanotechnology approaches have demonstrated the potential for intracellular drug delivery and drug delivery through Blood Brain Barrier. Though many progresses were reached in nanomedicine, still a conceptual understanding of biological responses to nanoparticles have to be developed for applying safe nanomaterial drug delivery in future.

\section{References}

1. Abd el-Razek NEE, Shoman SA, Mohamed AF (2011) Nanocapsulated Rift Valley Fever Vaccine Candidates and Relative Immunological and Histopathological Reactivity in Out Bred Swiss Mice. J Vaccines Vaccin 2: 115.

2. Pezzulo AA, Asif MH, Willander M, Zabner J (2011) In Situ Quantification of Glucose Concentration in Airway Surface Liquid With Functionalized ZnO Nanorod-Coated Microelectrodes. J Anal Bioanal Techniques S7: 002.

3. Rosen JE, Yoffe S, Meerasa A, Verma M, Gu FX (2011) Nanotechnology and Diagnostic Imaging: New Advances in Contrast Agent Technology. J Nanomedic Nanotechnol 2: 115.

4. Thomas S, Waterman P, Chen S, Marinelli B, Seaman M, et al. (2011) Development of Secreted Protein and Acidic and Rich in Cysteine (SPARC) Targeted Nanoparticles for the Prognostic Molecular Imaging of Metastatic Prostate Cancer. J Nanomedic Nanotechnol 2: 112.

5. Lukianova-Hleb EY, Oginsky AO, Shenefelt DL, Drezek RA, Hafner JH, et al. (2011) Rainbow Plasmonic Nanobubbles: Synergistic Activation of Gold Nanoparticle Clusters. J Nanomedic Nanotechnol 2: 104.

6. Kanwar JR, Zhou SF, Gurudevan S, Barrow CJ, Kanwar RK (2011) Toll Like Receptors Play a Role in General Immunity, Eye Infection and Inflammation: TIrs for Nanodelivery. J Clin Cell Immunol 2: 114

7. Li Y, Hu M, Qi B, Wang X, Du Y (2011) Preparation and Characterization 
of Biocompatible Quaternized Chitosan Nanoparticles Encapsulating CdS Quantum Dots. J Biotechnol Biomaterial 1: 108.

8. Yun Y, Conforti L, Muganda P, Sankar J (2011) Nanomedicine-based Synthetic Biology. J Nanomedic Biotherapeu Discover 1: 102e.

9. Knight LC, Romano JE, Krynska B, Faro S, Mohamed FB, et al. (2010) Binding and Internalization of Iron Oxide Nanoparticles Targeted To Nuclear Oncoprotein. J Mol Biomark Diagn 1: 102.

10. Lechleider R, Pastan I (2011) Advances in the Development of Anti-CD22 Immunotoxins Containing Pseudomonas Exotoxin for Treatment of Hematologic Malignancies. J Cancer Sci Ther 3: 50-52.

11. Caraglia M, Rosa GD, Abbruzzese A, Leonetti C (2011) Nanotechnologies: New Opportunities for Old Drugs. The Case of Aminobisphosphonates. J Nanomedic Biotherapeu Discover 1: 103e.

12. Nakamura J, Nakajima N, Matsumura K, Hyon SH (2011) In Vivo Cance Targeting of Water-Soluble Taxol by Folic Acid Immobilization. J Nanomedic Nanotechnol 2: 106.

13. Salim N, Basri M, Abd. Rahman MB, Abdullah DK, Basri H, et al. (2011) Phase Behaviour, Formation and Characterization of Palm-Based Esters Nanoemulsion Formulation containing Ibuprofen. J Nanomedic Nanotechnol 2: 113.

14. Pandurangappa C, Lakshminarasappa BN (2011) Optical absorption and Photoluminescence studies in Gamma-irradiated nanocrystalline CaF2. J Nanomedic Nanotechnol 2: 108

15. De Jong, W. H., \& Borm, P. J. A. (2008). Drug delivery and nanoparticles: applications and hazards. International journal of nanomedicine, 3: 133-49.

16. Vijaya Shanti B, Mrudula T, Naga Deepth CH, Sree Venkateshwarlu Y (2011) Novel Applications of Nanotechnology in Life Sciences. J Bioanal Biomed S11.

17. An NT, Dong NT, Hanh PTB, Nhi TTY, Vu DA, et al. (2010) SilverNcarboxymethyl Chitosan Nanocomposites: Synthesis and its Antibacterial Activities. J Bioterr Biodef 1: 102.

18. Nguyen KT (2011) Targeted Nanoparticles for Cancer Therapy: Promises and Challenges. J Nanomedic Nanotechnol 2: $103 \mathrm{e}$.

19. Nanjwade BK, Derkar GK, Bechra HM, Nanjwade VK, Manvi FV (2011) Design and Characterization of Nanocrystals of Lovastatin for Solubility and Dissolution Enhancement. J Nanomedic Nanotechnol 2: 107.

20. Mehrotra A, Nagarwal RC, Pandit JK (2010) Fabrication of Lomustine Loaded Chitosan Nanoparticles by Spray Drying and in Vitro Cytostatic Activity on Human Lung Cancer Cell Line L132. J Nanomedic Nanotechnolo 1: 103.

21. Lobo AO, Marciano FR, Regiani I, Matsushima JT, Ramos SC, et al. (2011) Influence of Temperature and Time For Direct Hydroxyapatite Electrodeposition on Superhydrophilic Vertically Aligned Carbon Nanotube Films. J Nanomedic Nanotechnol S8: 001.

22. Yan H, Mochizuki Y, Jo T, Okuzaki H (2011) Single-Walled-Carbon- NanotubeBased Field-Effect Transistors with Biosensing Functions for Prostate-SpecificAntigen. J Bioequiv Availab 3: 69-71.

23. Menaa B (2011) The Importance of Nanotechnology in Biomedical Sciences. $J$ Biotechnol Biomaterial 1: 105e.

24. Mizuno K, Zhiyentayev T, Huang L, Khalil S, Nasim F, et al. (2011) Antimicrobia Photodynamic Therapy with Functionalized Fullerenes: Quantitative Structureactivity Relationships. J Nanomedic Nanotechnol 2: 109.

25. Bareggi Renato NV, Paola N (2010) New Targeted Therapies Against Breast Cancer. J Carcinogene Mutagene 1: 110.

26. Kwangjae C, Xu W, Shuming N, Zhuo C, Dong MS (2008) Therapeutic Nanoparticles for Drug Delivery in Cancer, Clin Cancer Res 14: 1310

27. John I (2011) Nanotechnology-based Diagnostics; Are we Facing the Biotechnology Eevolution of the 21st Century? Mycobact Diseases 1: e102.

28. Rosarin FS, Mirunalini S (2011) Nobel Metallic Nanoparticles with Novel Biomedical Properties. J Bioanal Biomed 3: 85-91.

29. Zheng J, Clogston JD, Patri AK, Dobrovolskaia MA, McNeil SE (2011) Sterilization of Silver Nanoparticles Using Standard Gamma Irradiation Procedure Affects Particle Integrity and Biocompatibility. J Nanomedic Nanotechnol S5: 001.

30. Pezzulo AA, Asif MH, Willander M, Zabner J (2011) In Situ Quantification of
Glucose Concentration in Airway Surface Liquid With Functionalized ZnO Nanorod-Coated Microelectrodes. J Anal Bioanal Techniques S7:002

31. Douroumis D (2011) Mesoporous silica Nanoparticles as Drug Delivery System. J Nanomedic Nanotechnol 2: 102e.

32. Patil A, Chirmade UN, Trivedi V, Lamprou DA, Urquhart A, Douroumis D (2011) Encapsulation of Water Insoluble Drugs in Mesoporous Silica Nanoparticles using Supercritical Carbon Dioxide. J Nanomedic Nanotechnol 2: 111

33. Mera A, Araki J, Ohtsuki T, Shimosaka M, Yoshida N (2011) Chitin Nanowhiskers Mediate Transformation of Escherichia coli by Exogenous Plasmid DNA. J Biotechnol Biomaterial 1: 114

34. Anwunobi AP, Emeje MO (2011) Recent Applications of Natural Polymers in Nanodrug Delivery. J Nanomedic Nanotechnol S4: 002.

35. Elgindy N, Elkhodairy K, Molokhia A, ElZoghby A (2011) Biopolymeric Nanoparticles for Oral Protein Delivery: Design and In Vitro Evaluation. Nanomedic Nanotechnol 2: 110

36. Sudhakar A (2009) History of Cancer, Ancient and Modern Treatment Methods. J Cancer Sci Ther 1: i-iv.

37. Yiyao L, Hirokazu M and Michihiro N (2007) Nanomedicine for drug delivery and imaging: A promising avenue for cancer therapy and diagnosis using targeted functional nanoparticles, Int J Cancer: 120: 2527-2537.

38. Saboktakin MR, Tabatabaie RM, Maharramov A, Ramazanov MA (2011) Synthesis and Characterization of Biodegradable Thiolated Chitosan Nanoparticles as Targeted Drug Delivery System. J Nanomedic Nanotechnol S4: 001

39. Asif MH, Elinder F, Willander M (2011) Electrochemical Biosensors Based on $\mathrm{ZnO}$ Nanostructures to Measure Intracellular Metal Ions and Glucose. J Anal Bioanal Techniques S7: 003

40. Sun CZ, Lu CT, Zhao YZ, Guo P, Tian JL, et al. (2011) Characterization of the Doxorubicin-Pluronic F68 Conjugate Micelles and Their Effect on Doxorubicin Resistant Human Erythroleukemic Cancer Cells. J Nanomedic Nanotechnol 2: 114

41. Tsigelny IF, Simberg D (2011) Has the Time for In silico Design of Nanomedicines Finally Arrived? J Nanomedic Biotherapeu Discover 1: 104e.

42. Chou LYT, Ming K, Chan WCW (2011) Strategies for the intracellular delivery of nanoparticles. Chem Soc Rev 40: 233-245.

43. Huang X, Kang B, Qian W, Mackey MA, Chen PC (2010) Comparative study of photothermolysis of cancer cells with nuclear-targeted or cytoplasm-targeted gold nanospheres: continuous wave or pulsed lasers, J. Biomed Opt 15 058002

44. Goldberg M, Langer R, Jia X (2007) Nanostructured materials for applications in drug delivery and tissue engineering, J Biomater Sci Polym Ed. 18: 241-268.

45. Rajendran L, Knölker HJ, Simons K (2010) Subcellular targeting strategies for drug design and delivery. Nat Rev Drug Discov 9: 29-42.

46. Yamada Y, Akita H, Kogure K, Kamiya H, Harashima H (2007) Mitochondria drug delivery and mitochondrial disease therapy--an approach to liposomebased delivery targeted to mitochondria. Mitochondrion, 7: 63-71.

47. Zhao Y, Haney MJ, Mahajan V, Reiner BC, Dunaevsky A, et al. (2011) Active Targeted Macrophage-mediated Delivery of Catalase to Affected Brain Regions in Models of Parkinson's Disease. J Nanomedic Nanotechnol S4: 003.

48. Juillerat-Jeanneret $L$ (2008) The targetted delivery of cancer drugs across the Blood Brain Barrier: Chemical modification of drugs or drug nanoparticles. Drug Discov Today 13: 1099-1106.

49. Ringe K, Walz CM, Sabel BA (2004) Nanoparticle Drug Delivery to the Brain, Encyclopedia of Nanoscience and Nanotechnology 7: 91-104.

50. Lin LN, Liu Q, Song L, Liu FF, Sha JX (2010) Recent advances in nanotechnology based drug delivery to the brain. Cytotechnology 62: 377-380.

51. Venkatraman SS, Ma LL, Natarajan JV, Chattopadhyay S (2010) Polymer- and liposome-based nanoparticles in targeted drug delivery. Front Biosci (Schol Ed) 2: 801-814.

52. Shakeel F, Ramadan W, Shafiq S (2009) Solubility and Dissolution Improvemen of Aceclofenac using Different Nanocarriers. J Bioequiv Availab 1: 39-043.

53. Al-Jamal, W. T., \& Kostarelos, K. (2011). Liposomes: From a Clinically Established Drug Delivery System to a Nanoparticle Platform for Theranostic 
Nanomedicine. Accounts of Chemical Research, XXX(Xx), null-null. American Chemical Society.

54. Park JW(2002) Liposome-based drug delivery in breast cancer treatment Breast Cancer Research 4: 95-99.

55. Dibirdik I, Yiv S, Qazi S, Uckun FM (2010) In vivo Anti-Cancer Activity of a Liposomal Nanoparticle Construct of Multifunctional Tyrosine Kinase Inhibitor 4-(4'-Hydroxyphenyl)-Amino-6,7 Dimethoxyquinazoline. J Nanomedic Nanotechnolo 1: 101

56. Eshita Y, Higashihara J, Onishi M, Mizuno M, Yoshida J, et al. (2011) Mechanism of the Introduction of Exogenous Genes into Cultured Cells Using DEAE-Dextran-MMA Graft Copolymer as a Non-Viral Gene Carrier. II. Its Thixotropy Property. J Nanomedic Nanotechnol 2: 105.

57. Ringe K, Walz CM, Sabel BA (2004) Nanoparticle Drug Delivery to the Brain Encyclopedia of Nanoscience and Nanotechnology 7: 91-104

58. Shih MF, Wu CH, Cherng JY (2011) Bioeffects of Transient and LowIntensity Ultrasound on Nanoparticles for a Safe and Efficient DNA Delivery. J Nanomedic Nanotechnol S3: 001.

59. Tarl Prow, Jacob N. Smith, Rhonda Grebe, Jose H. Salazar, Nan Wang, et al Construction, gene delivery, and expression of DNA tethered Nanoparticles, Molecular Vision 2006; 12: 606-615.
60. Vellaichamy A, Lin CY, Aye TT, Kunde GR, Nesvizhskii Al, et al. (2010) A Chloroform-Assisted Protein Isolation Method Followed by Capillary NanoLCMS Identify Estrogen-Regulated Proteins from MCF7 Cells. J Proteomics Bioinform 3: 212-220.

61. Pandurangappa C, Lakshminarasappa BN (2011) Optical absorption and Photoluminescence studies in Gamma-irradiated nanocrystalline CaF2. J Nanomedic Nanotechnol 2: 108.

62. Abu Shawish HM, Al-Dalou AR, Abu Ghalwa N, Abou Assi AA (2010) Potentiometric Sensor for Determination of Tramadol Hydrochloride in Pharmaceutical Preparations and Biological Fluids. Pharm Anal Acta 1: 103.

63. Tateishi A, Cauchi M, Tanoue C, Migita S, Coleman SK, et al. (2011) Discerning Data Analysis Methods to Clarify Agonistic/Antagonistic Actions on the lon Flux Assay of Ligand-Gated Ionotropic Glutamate Receptor on Engineered PostSynapse Model Cells. J Biosens Bioelectron 2: 104.

64. Achyuthan K (2011) Whither Commercial Nanobiosensors? J Biosens Bioelectron 2: 102e.

65. Pandey RR, Saini KK, Dhayal M (2010) Using Nano-Arrayed Structures in SolGel Derived Mn2+ Doped Tio2 for High Sensitivity Urea Biosensor. J Biosens Bioelectron 1: 101 